\title{
openheart Statin treatment and LDL-cholesterol treatment goal attainment among individuals with familial hypercholesterolaemia in primary care
}

\author{
Barbara lyen (i) , Ralph K Akyea, Stephen Weng (D) , Joe Kai (D),
} Nadeem Qureshi (D)

- Additional supplemental material is published online only. To view, please visit the journal online (http://dx.doi.org/10. 1136/openhrt-2021-001817).

To cite: Iyen B, Akyea RK, Weng $\mathrm{S}$, et al. Statin treatment and LDL-cholesterol treatment goal attainment among individuals with familial hypercholesterolaemia in primary care. Open Heart 2021;8:e001817. doi:10.1136/ openhrt-2021-001817

JK and NQ contributed equally.

Received 13 September 2021 Accepted 6 October 2021

Check for updates

(c) Author(s) (or their employer(s)) 2021. Re-use permitted under CC BY. Published by BMJ.

Primary Care Stratified Medicine, Population Health and Lifespan Sciences, University of Nottingham Faculty of Medicine and Health Sciences, Nottingham, UK

Correspondence to Dr Barbara lyen; barbara.iyen@ nottingham.ac.uk

\section{ABSTRACT}

Objectives Guidance recommends statin treatment in familial hypercholesterolaemia (FH) to achieve at least a $50 \%$ reduction in low-density lipoprotein cholesterol $(\mathrm{LDL}-\mathrm{C})$. We assessed statin prescribing rates and LDL-C treatment goal attainment among individuals with $\mathrm{FH}$ in primary care.

Methods Using primary care electronic health records from the UK Clinical Practice Research Datalink, we identified adults with recorded diagnosis of $\mathrm{FH}$, statin treatment and measures of LDL-C prior to (baseline) and 12 months after initiating statin treatment. The percentage change in LDL-C was determined, and then baseline and treatment characteristics were assessed by LDL-C treatment goal attainment.

Results 0 f 3064 adults (mean age 50.8 years) with recorded diagnosis of $\mathrm{FH}$ and repeat LDL-C measures, $50 \%$ reduction in LDL-C from baseline was attained in 895 individuals (29.2\%) in 12 months. Compared with those who did not attain this goal, these people were predominantly women; they were older at time of $\mathrm{FH}$ diagnosis (53.4 years vs 49.7 years) and first statin treatment ( 53.2 years vs 49.2 years) and had higher pretreatment total cholesterol (8.20 (SD 1.38) $\mathrm{mmol} / \mathrm{L}$ vs 7.57 (SD 1.39) $\mathrm{mmol} / \mathrm{L})$ and pretreatment LDL-C (5.83 (SD 1.36) mmol/L vs 5.25 (SD 1.40) mmol/L). A higher proportion of individuals who attained the treatment goal was prescribed high-potency and medium-potency statins ( $24.3 \%$ and $71.7 \%$ vs $20.2 \%$ and $69.3 \%$, respectively). Conclusions Less than a third of individuals on statin treatment for $\mathrm{FH}$ in the community achieve recommended reductions in LDL-C. Greater awareness and optimisation of treatment for FH using higher-potency statins are needed.

\section{INTRODUCTION}

Familial hypercholesterolaemia $(\mathrm{FH})$ is a genetic condition that causes lifelong elevated low-density lipoprotein cholesterol (LDL-C) and is associated with significant cardiovascular morbidity and mortality. ${ }^{12}$ Reducing the LDL-C level is one of the primary goals in managing individuals with $\mathrm{FH}$, and treatment with lipid-lowering drugs such as statins

\section{Key questions}

What is already known about this subject?

- The use of statins to reducing low-density lipoprotein cholesterol (LDL-C) burden is one of the central goals of familial hypercholesterolaemia (FH) management, and national and international guidance recommend that treatment should achieve at least a $50 \%$ reduction in LDL-C from baseline measurement

What does this study add?

- This study provides novel evidence of suboptimal LDL-C reduction in individuals with $\mathrm{FH}$ who are being treated with statins, such that less than $40 \%$ of patients are treated with high-intensity statins at 12 months, and two-thirds of patients initiated on statin treatment fail to attain the recommended treatment target.

How might this impact on clinical practice?

- These findings highlight the need for greater awareness of $\mathrm{FH}$ and the need to optimally manage these patients with high-intensity statins.

reduces LDL-C burden and consequently the cardiovascular disease (CVD) and mortality risk associated with $\mathrm{FH}^{34}$

In the treatment of adults with $\mathrm{FH}$, the UK National Institute for Health and Care Excellence (NICE) guidelines recommend at least a $50 \%$ reduction in serum LDL-C concentration from the baseline measurement. ${ }^{5}$ Recent guidelines from the European Society of Cardiology/European Atherosclerosis Society (ESC/EAS) recommend in addition to the LDL-C reduction of $\geq 50 \%$ from baseline, LDL-C treatment targets of $<1.8$ $\mathrm{mmol} / \mathrm{L}$ in adults with $\mathrm{FH}$, or $<1.4 \mathrm{mmol} / \mathrm{L}$ in adults with $\mathrm{FH}$ and CVD or other major risk factors. ${ }^{6}$

The degree of LDL-C reduction varies with statins of different potencies. ${ }^{7}$ Also, there is interindividual variation in LDL-C reduction 
with statin therapy, ${ }^{8}$ which may partly be due to poor medication compliance ${ }^{9}$ or genetic factors. ${ }^{10}$ In the general population, it has been shown that over half of patients commenced on statin therapy for primary prevention of CVD do not attain optimal LDL-C reduction after 24 months of treatment. ${ }^{11}$ There is limited evidence of the magnitude of LDL-C reduction after initiation of statins in individuals with $\mathrm{FH}$, in real-world community settings. This study explored statin prescribing patterns in individuals with $\mathrm{FH}$ in primary care and then assessed LDL-C reduction and treatment goal attainment in these patients following initiation of statins.

\section{METHODS}

\section{Data source}

The UK Clinical Practice Research Datalink (CPRD) is a large electronic database of anonymised longitudinal routine primary care health records. The database has coverage of approximately $15 \%$ of the UK population, and patients are representative of the UK population in terms of age and sex. ${ }^{12}$ Individuals' primary care records in CPRD were linked with their secondary care records (hospital episode statistics) and death registration records from the Office for National Statistics. Data access and ethical approval for the study were granted by the CPRD Independent Scientific Advisory Committee (protocol number 20_093) in June 2020.

\section{Study population}

We identified all individuals aged 18 years or older who had a coded clinical diagnosis of $\mathrm{FH}$ in their primary care record, a record of treatment with statins of known potency, a measure of LDL-C prior to commencing statin treatment and a repeat LDL-C measure 12 months after initiating statin treatment. Patients were eligible for inclusion in the study if they had been registered in their general practice for at least 1 year and had no records of pre-existing CVD before being commenced on statins.

\section{LDL-C measures and other covariates}

The baseline LDL-C measure was defined as the most recent LDL-C in patients' records in the 12-month period before the first treatment with statins. LDL-C at 12 months was the last measure recorded after 6 months and within the 12 months after initiating statin treatment.

Patient characteristics collected at baseline include age; sex; ethnicity; history of alcohol misuse; smoking status; physical activity level; comorbidities including atrial fibrillation, hypertension, type 2 diabetes, overweight/ obesity and chronic kidney disease; and use of medication that could secondarily cause hyperlipidaemia such as corticosteroids and antipsychotics. The categorising of statin potency (high, medium or low intensity) takes account of the estimated percentage reduction in LDL-C. expected using varying doses of different statins. ${ }^{13}$ Low-intensity statins produce an LDL-C reduction of 20\%-30\%, medium-intensity statins produce a reduction of $31 \%-40 \%$ and high-intensity statins produce an LDL-C reduction greater than $40 \%^{7}$

\section{Outcome ascertainment}

Patients were followed up from the first date of prescription of a statin with known potency. Baseline lipid measures (LDL-C) and repeated LDL-C measure at 12 months were used to determine the percentage change in LDL-C during the period of follow-up. Based on the NICE FH treatment guideline recommendation that states that statin treatment in individuals with $\mathrm{FH}$ should aim to achieve at least a $50 \%$ reduction in LDL-C concentration from baseline measurement, ${ }^{14}$ patients were classified as goal attainers or non-goal attainers, depending on whether they achieved the $50 \%$ or greater reduction in LDL-C or not, respectively.

\section{Statistical analyses}

In the study cohort of individuals with $\mathrm{FH}$, serum concentrations of lipids such as total cholesterol, LDL-C, highdensity lipoprotein cholesterol and triglycerides, as well as types and potency of lipid-lowering treatment, were assessed at baseline, 12 months and 24 months. Change in LDL-C at 12 months was calculated by dividing the difference between the LDL-C at baseline and LDL-C at 12 months, by the baseline LDL-C concentration, and this was expressed as a percentage. As previously described, individuals were categorised as goal attainers or non-goal attainers, depending on their percentage change in LDL-C at 12 months. Baseline characteristics including the clinical profile and treatment characteristics were explored in the cohort who attained the $50 \%$ LDL-C reduction treatment goal and those who did not. $\chi^{2}$ test of significance, t-tests and Mann-Whitney $\mathrm{U}$ tests were used to assess differences in categorical and continuous variables between LDL-C goal attainers and non-goal attainers, depending on the distribution of the variables. After determining the change in LDL-C at 12 months, further analyses determined change in LDL-C at 24 months. LDL-C at 24 months was defined as the last LDL-C measure after 12 months but within the 24-month period after initiating statin treatment. We further assessed attainment of at least a $40 \%$ reduction in LDL-C, which is the NICE-recommended target for the general population of non-FH individuals who are initiated on statins for the primary prevention of CVD. ${ }^{15}$

Following primary analyses conducted on all individuals with $\mathrm{FH}$ diagnosis in primary care, a sensitivity analysis was done on the subset of individuals who had a diagnosis of $\mathrm{FH}$ as well as baseline total cholesterol of $7.5 \mathrm{mmol} / \mathrm{L}$ or greater, which is the $\mathrm{FH}$ total cholesterol diagnostic threshold. ${ }^{2}$ Further sensitivity analysis also assessed treatment goal attainment with respect to the ESC/EAS guidelines. ${ }^{6}$ Statistical significance was defined at the $\mathrm{p}<0.05$ level. Analyses were conducted using Stata SE V.16. 
Table 1 Lipid profile, statin treatment and treatment goal attainment in adults with FH in UK primary care $(n=3064)$

\begin{tabular}{|c|c|c|c|c|}
\hline Characteristics & Unit & Baseline & 12 months & 24 months \\
\hline \multicolumn{5}{|l|}{ Individuals' lipid profile } \\
\hline Total cholesterol (mmol/L) & Mean (SD) & $7.76(1.42)$ & $5.56(1.40)$ & $5.42(1.30)$ \\
\hline LDL-cholesterol (mmol/L) & Mean (SD) & $5.42(1.41)$ & $3.45(1.37)$ & $3.28(1.23)$ \\
\hline HDL-cholesterol (mmol/L) & Mean (SD) & $1.50(0.49)$ & $1.49(0.47)$ & $1.51(0.49)$ \\
\hline Triglycerides (mmol/L) & Median (IQR) & $1.70(1.20-2.47)$ & $1.40(1.00-2.00)$ & $1.32(0.93-2.00)$ \\
\hline \multicolumn{5}{|l|}{ Potency of prescribed statins* } \\
\hline & & $(n=3064)$ & $(n=2142)$ & $(n=1940)$ \\
\hline Low & $\mathrm{n}(\%)$ & 264 (8.62) & $142(6.63)$ & $111(5.72)$ \\
\hline Medium & & $2145(70.01)$ & $1225(57.19)$ & $1067(55.00)$ \\
\hline High & & $655(21.38)$ & $775(36.18)$ & $762(39.28)$ \\
\hline \multicolumn{5}{|l|}{ LDL-C reduction at follow-up } \\
\hline FH treatment goal attainment & & & $(n=3064)$ & $(n=1662)$ \\
\hline Attained $\geq 50 \%$ reduction & $\mathrm{n}(\%)$ & & $895(29.21)$ & $558(33.57)$ \\
\hline Non-attainment of $50 \%$ reduction & & & $2169(70.79)$ & $1104(66.43)$ \\
\hline General population goal attainment & & & $(n=3064)$ & $(n=1662)$ \\
\hline Attained $\geq 40 \%$ reduction & $\mathrm{n}(\%)$ & & $1566(51.11)$ & $935(56.26)$ \\
\hline Non-attainment of $40 \%$ reduction & & & $1498(48.89)$ & 727 (43.74) \\
\hline
\end{tabular}

*Statin potency at 12 months and 24 months was reported in individuals with LDL-C records at baseline and 12 months, as well as statin potency records at 12 and 24 months, respectively. Total number and percentage $\mathrm{n}(\%)$ for potency were based only on those with potency records, with the exclusion of missing records.

$\mathrm{FH}$, familial hypercholesterolaemia; HDL, high-density lipoprotein; LDL, low-density lipoprotein.

\section{RESULTS}

A total of 3064 adults had clinical diagnostic codes for $\mathrm{FH}$, records of statin with known potency at baseline, LDL-C measures at baseline and 12 months and no history of CVD prior to being initiated on statins, between December 1988 and August 2020. Statin treatment and lipid profile of these individuals at baseline, as well as 12 and 24 months after starting treatment with statins, are shown in table 1.

Over the period of follow-up, the proportion of individuals prescribed with low-potency statins reduced from baseline to 24 months $(8.6 \%$ at baseline, $6.6 \%$ at 12 months and $5.7 \%$ at 24 months), while the proportion prescribed with high-potency statins increased over time, from $21.4 \%$ at baseline, $36.2 \%$ at 12 months and $39.3 \%$ at 24 months. Compared with the baseline LDL-C value, there was a mean reduction in LDL-C of $36.3 \%$ at 12 months $(5.42 \mathrm{mmol} / \mathrm{L}$ vs $3.45 \mathrm{mmol} / \mathrm{L})$ and $39.5 \%$ at 24 months $(5.42 \mathrm{mmol} / \mathrm{L}$ vs $3.28 \mathrm{mmol} / \mathrm{L})$.

Prior to restricting our study sample to the 3064 eligible adults with FH (who were treated with statins, with no CVD prior to statin initiation and with LDL-C measures at baseline and 12 months), we assessed the pattern of lipid-lowering treatment prescribing 8234 adults with FH and no prior CVD. Findings showed an increase in the prescribing of non-statin lipid-lowering treatments such as ezetimibe (monotherapy or prescribed in combination with statins) $(0.05 \%$ at baseline, $3.2 \%$ at 12 months and $4.2 \%$ at 24 months) and fibrates $(0 \%$ at baseline, $1.1 \%$ at
12 months and $1.4 \%$ at 24 months) (online supplemental table 1).

\section{LDL-C treatment goal attainment}

Of the 3064 individuals in our FH study cohort, 895 $(29.2 \%)$ achieved a $50 \%$ or greater reduction in LDL-C concentration from the baseline measure, 12 months after statin initiation. Repeat LDL-C measures were available for 1662 individuals at 24 months, and 558 (33.6\%) achieved a $50 \%$ or greater reduction in LDL-C from baseline (table 1).

A $40 \%$ reduction in LDC-C from baseline, which is the NICE-recommended goal for the general population of non-FH individuals initiated on statins for primary prevention of CVD, was achieved at 12 months by $51 \%$ of the $\mathrm{FH}$ cohort and at 24 months by $56.3 \%$ of the cohort.

\section{Characteristics of individuals by LDL treatment goal attainment at 12 months}

Table 2 shows the baseline and lipid-lowering treatment characteristics of individuals, by LDL treatment goal attainment at 12 months. Compared with individuals who did not attain the LDL-C treatment goal of $50 \%$ or greater, a significantly higher proportion of those who attained the goal was women. Individuals who attained the treatment goal were of older age at time of FH diagnosis (53.4 years vs 49.7 years) and at time of first statin treatment ( 53.2 years vs 49.2 years), and they had significantly higher mean pretreatment total cholesterol (8.20 (SD 
Table 2 Baseline and treatment characteristics of individuals, by LDL-cholesterol treatment goal attainment at 12 months $(n=3064)$

\begin{tabular}{|c|c|c|c|c|c|}
\hline \multirow{2}{*}{$\begin{array}{l}\text { Patient or treatment } \\
\text { characteristics }\end{array}$} & \multirow[b]{2}{*}{ Unit } & \multirow{2}{*}{$\begin{array}{l}\text { Total N (\%) } \\
3064(100)\end{array}$} & \multirow{2}{*}{$\begin{array}{l}\text { LDL-C goal attained } \\
\text { N (\%) } \\
895(29.21)\end{array}$} & \multirow{2}{*}{$\begin{array}{l}\text { LDL-C goal not } \\
\text { attained N (\%) } \\
2169(70.79)\end{array}$} & \multirow[b]{2}{*}{$P$ value } \\
\hline & & & & & \\
\hline Women & $\mathrm{N}(\%)$ & $1873(61.13)$ & 590 (65.92) & $1283(59.15)$ & $<0.001$ \\
\hline Ethnicity & $\mathrm{N}(\%)$ & & & & \\
\hline White & & 1610 (91.74) & 452 (92.24) & 1158 (91.54) & \\
\hline Asian/British Asian & & $74(4.22)$ & $10(4.08)$ & $54(4.27)$ & 0.366 \\
\hline Black/Black British & & $27(1.54)$ & $7(1.43)$ & $20(1.58)$ & \\
\hline Mixed & & $10(0.57)$ & 0 & $10(0.79)$ & \\
\hline Others & & $34(1.94)$ & $11(2.24)$ & $23(1.83)$ & \\
\hline Age (years) at FH diagnosis & Mean (SD) & $50.79(13.13)$ & $53.44(12.60)$ & $49.71(13.20)$ & $<0.001$ \\
\hline Age (years) at first statin & Mean (SD) & $50.33(12.83)$ & $53.18(12.48)$ & $49.16(12.80)$ & $<0.001$ \\
\hline $\begin{array}{l}\text { Pretreatment total cholesterol } \\
(\mathrm{mmol} / \mathrm{L})\end{array}$ & Mean (SD) & $7.76(1.42)$ & $8.20(1.38)$ & 7.57 (1.39) & $<0.001$ \\
\hline Pretreatment LDL-C (mmol/L) & Mean (SD) & $5.42(1.41)$ & $5.83(1.36)$ & $5.25(1.40)$ & $<0.001$ \\
\hline $\begin{array}{l}\text { Post-treatment LDL-C (12 months) } \\
\text { (mmol/L) }\end{array}$ & Mean (SD) & $3.45(1.37)$ & $2.43(0.63)$ & $3.88(1.37)$ & $<0.001$ \\
\hline Pretreatment HDL-C (mmo//L) & Mean (SD) & $1.50(0.49)$ & $1.59(0.57)$ & $1.46(0.45)$ & $<0.001$ \\
\hline Pretreatment triglycerides $(\mathrm{mmol} / \mathrm{L})$ & Mean (SD) & $1.70(1.20-2.47)$ & $1.70(1.20-2.47)$ & $1.70(1.20-2.46)$ & 0.5087 \\
\hline Statin potency at baseline & $\mathrm{N}(\%)$ & & & & \\
\hline Low & & $264(8.62)$ & $36(4.02)$ & $225(10.51)$ & \\
\hline Medium & & $2145(70.01)$ & $642(71.73)$ & $1503(69.29)$ & $<0.001$ \\
\hline High & & $655(21.38)$ & $217(24.25)$ & $438(20.19)$ & \\
\hline Statin potency at 6 months & $\mathrm{N}(\%)$ & & & & \\
\hline Low & & $188(6.14)$ & $26(2.91)$ & $162(7.47)$ & \\
\hline Medium & & $1571(51.27)$ & $485(54.19)$ & $1086(50.07)$ & $<0.0001$ \\
\hline High & & $772(25.20)$ & $298(33.30)$ & $474(21.85)$ & \\
\hline Missing record & & $533(17.40)$ & $86(9.61)$ & 447 (20.61) & \\
\hline Statin potency at 12 months & $\mathrm{N}(\%)$ & & & & \\
\hline Low & & $142(4.63)$ & $17(1.90)$ & $125(5.76)$ & $<0.0001$ \\
\hline Medium & & $1225(39.98)$ & $426(47.60)$ & 799 (36.84) & \\
\hline High & & 775 (25.29) & 301 (33.63) & $474(21.85)$ & \\
\hline Missing record & & $922(30.09)$ & $151(16.87)$ & $771(35.55)$ & \\
\hline Cigarette smoking status at baseline & $\mathrm{N}(\%)$ & $n=2126$ & $\mathrm{n}=615$ & $n=1511$ & \\
\hline Current & & $628(29.54)$ & $160(26.02)$ & $468(30.97)$ & $<0.001$ \\
\hline Ex & & $461(21.68)$ & $135(21.95)$ & $326(21.58)$ & \\
\hline Never & & $1037(48.78)$ & $320(52.03)$ & $717(47.45)$ & \\
\hline Alcohol misuse (yes) & $\mathrm{N}(\%)$ & $35(1.14)$ & $14(1.56)$ & $21(0.97)$ & 0.158 \\
\hline Atrial fibrillation & $\mathrm{N}(\%)$ & $18(0.59)$ & $8(0.89)$ & $10(0.46)$ & 0.154 \\
\hline Chronic kidney disease & $\mathrm{N}(\%)$ & $40(1.31)$ & $19(2.12)$ & $21(0.97)$ & 0.01 \\
\hline Hypertension & $\mathrm{N}(\%)$ & $406(13.25)$ & $120(13.41)$ & $286(13.19)$ & 0.869 \\
\hline Type 2 diabetes & $\mathrm{N}(\%)$ & $45(1.47)$ & $15(1.68)$ & $30(1.38)$ & 0.54 \\
\hline Obesity/overweight & $\mathrm{N}(\%)$ & $320(10.44)$ & $97(10.84)$ & $223(10.28)$ & 0.647 \\
\hline Physical activity level & $\mathrm{N}(\%)$ & $\mathrm{n}=331$ & $\mathrm{n}=94$ & $\mathrm{n}=237$ & \\
\hline Extremely inactive & & $23(6.95)$ & $7(7.45)$ & $16(6.75)$ & 0.54 \\
\hline Sedentary & & $29(8.76)$ & $7(7.45)$ & $22(9.28)$ & \\
\hline
\end{tabular}


Table 2 Continued

\begin{tabular}{|c|c|c|c|c|c|}
\hline \multirow{2}{*}{$\begin{array}{l}\text { Patient or treatment } \\
\text { characteristics }\end{array}$} & \multirow[b]{2}{*}{ Unit } & \multirow{2}{*}{$\begin{array}{l}\text { Total N (\%) } \\
3064(100)\end{array}$} & \multirow{2}{*}{$\begin{array}{l}\text { LDL-C goal attained } \\
\text { N (\%) } \\
895(29.21)\end{array}$} & \multirow{2}{*}{$\begin{array}{l}\text { LDL-C goal not } \\
\text { attained N (\%) } \\
2169(70.79) \\
\end{array}$} & \multirow[b]{2}{*}{$P$ value } \\
\hline & & & & & \\
\hline Moderately active & & $271(81.87)$ & 76 (80.85) & $195(82.28)$ & \\
\hline Extremely active & & $8(2.42)$ & $4(4.26)$ & $4(1.69)$ & \\
\hline Use of other medications & $\mathrm{N}(\%)$ & & & & \\
\hline Antipsychotics & & $418(13.64)$ & $148(16.54)$ & $270(12.45)$ & 0.003 \\
\hline Corticosteroids & & $292(9.53)$ & $108(12.07)$ & $184(8.48)$ & 0.002 \\
\hline
\end{tabular}

FH, familial hypercholesterolaemia; HDL, high-density lipoprotein; LDL, low-density lipoprotein.

$1.38) \mathrm{mmol} / \mathrm{L}$ vs 7.57 (SD 1.39) $\mathrm{mmol} / \mathrm{L}$ ) and pretreatment LDL-C (5.83 (SD 1.36) mmol/L vs 5.25 (SD 1.40) $\mathrm{mmol} / \mathrm{L})$ than those who did not attain the treatment goal. Overall, the proportion of individuals treated with low-intensity and medium-intensity statins reduced, while the proportion of prescribed with high-intensity statins increased from baseline to 12 months. Significantly more people who attained the LDL-C treatment goal were treated with medium-intensity and high-intensity statins than those who did not attain the treatment goal, at baseline $(71.7 \%$ and $24.3 \%$ vs $69.3 \%$ and $20.2 \%$, respectively), 6 months $(54.2 \%$ and $33.3 \%$ vs $50.1 \%$ and $21.9 \%$, respectively) and 12 months (47.6\% and $33.6 \%$ vs $36.8 \%$ and $21.9 \%$, respectively) after statin initiation (table 2 and figure 1). Compared with individuals who attained the treatment goal, significantly higher proportions of those who did not attain the treatment goal were smokers at the time of their first statin treatment $(31.0 \%$ vs $26.0 \%)$. There were no significant differences in ethnicity, mean pretreatment triglycerides, alcohol consumption and prevalence of comorbidities such as atrial fibrillation, hypertension, type 2 diabetes, obesity and physical activity levels between those who attained a $\geq 50 \%$ LDL-C target and those who did not. The prevalence of chronic kidney disease was higher in those who attained the treatment goal compared with those who did not $(2.1 \%$ vs $1.0 \%)$, and more people who attained the treatment goal were on antipsychotics and corticosteroids than in those who did attain the treatment goal.

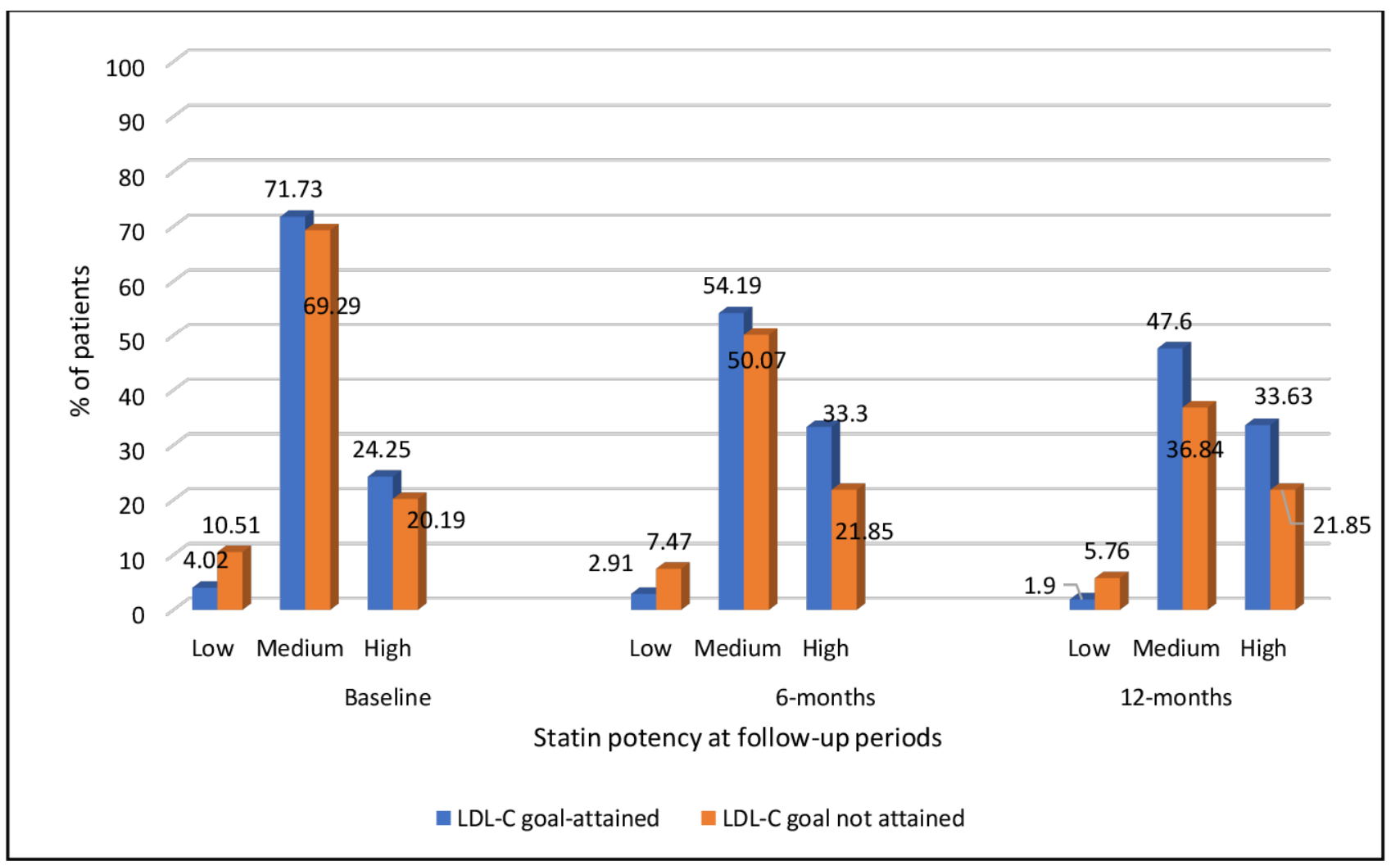

Figure 1 Prescribing of low-potency, medium-potency and high-potency statins among LDL-C goal attainers and non-goal attainers, at time of statin initiation (baseline) and 6 months and 12 months after statin initiation. LDL-C, low-density lipoprotein cholesterol. 
Table 3 Lipid profile at baseline, lipid-lowering treatment and treatment goal attainment, in sensitivity analyses restricted to adults with $\mathrm{FH}$ diagnosis and baseline total cholesterol $\geq 7.5 \mathrm{mmo} / \mathrm{L}$ ( $\mathrm{n}=1805$ at baseline)

\begin{tabular}{|c|c|c|c|c|}
\hline & & Baseline & 12 months & 24 months \\
\hline Total cholesterol (mmol/L) & Mean (SD) & $8.62(1.09)$ & $5.87(1.47)$ & $5.68(1.33)$ \\
\hline LDL-cholesterol (mmol/L) & Mean (SD) & $6.16(1.25)$ & $3.71(1.45)$ & $3.51(1.29)$ \\
\hline HDL-cholesterol (mmol/L) & Mean (SD) & $1.53(0.49)$ & $1.52(0.48)$ & $1.53(0.46)$ \\
\hline Triglycerides (mmol/L) & Median (IQR) & $1.80(1.29-2.65)$ & $1.40(1.00-2.10)$ & $1.39(1.00-2.00)$ \\
\hline Potency of prescribed statins* & $\mathrm{N}(\%)$ & $(n=1805)$ & $(n=1299)$ & $(n=1172)$ \\
\hline Low & & $144(7.98)$ & $84(6.47)$ & $56(4.78)$ \\
\hline Medium & & $1231(68.20)$ & $668(51.42)$ & $572(48.81)$ \\
\hline High & & $430(23.82)$ & $547(42.11)$ & $544(46.42)$ \\
\hline LDL-C reduction at time of follow-up & $\mathrm{N}(\%)$ & & $(n=1805)$ & $(n=982)$ \\
\hline Attained $50 \%$ reduction & & & 649 (35.96) & $423(43.08)$ \\
\hline Non-attained $50 \%$ reduction & & & $1156(64.04)$ & 559 (56.92) \\
\hline
\end{tabular}

*Statin potency at 12 months and 24 months was reported in individuals with records of LDL-C at 12 months, as well as records of statin potency at 12 and 24 months, respectively.

$\mathrm{FH}$, familial hypercholesterolaemia; HDL, high-density lipoprotein; LDL, low-density lipoprotein.

\section{Sensitivity analyses}

Table 3 shows the result of analyses restricted to 1805 individuals who had clinical FH diagnoses and baseline total cholesterol of $7.5 \mathrm{mmol} / \mathrm{L}$ or greater. Higher proportions of these individuals were treated with high-potency statins at baseline and 12 and 24 months, compared with the entire study cohort. Compared with the LDL-C concentration at baseline, there was a mean reduction in LDL-C of $39.8 \%$ at 12 months $(6.16 \mathrm{mmol} / \mathrm{L}$ vs 3.71 $\mathrm{mmol} / \mathrm{L})$ and $43.0 \%$ at 24 months $(6.16 \mathrm{mmol} / \mathrm{L}$ vs 3.51 $\mathrm{mmol} / \mathrm{L}$ ), which were greater than reductions observed in the entire cohort. Also, compared with the entire study cohort, a greater proportion of these individuals attained the NICE guideline-recommended LDL-C reduction of $50 \%$ or greater at 12 months $(36.0 \%$ vs $29.2 \%)$ and 24 months $(43.1 \%$ vs $33.6 \%)$.

Lastly, assessment of the LDL-C treatment goal attainment with respect to the ESC/EAS guidelines showed that only $4.5 \%$ of the study cohort achieved the goal of $50 \%$ LDL-C reduction and LDL-C $<1.8 \mathrm{mmol} / \mathrm{L}$ (online supplemental table 2). Further restrictions to the subset of individuals with clinical FH diagnoses and a diagnostic threshold of total cholesterol $\geq 7.5 \mathrm{mmol} / \mathrm{L}$ did not demonstrate an increase in the proportions who attained the ESC/EAS treatment goals.

\section{DISCUSSION}

In this large prospective cohort of adults with $\mathrm{FH}$, the NICE-recommended treatment goal of at least a $50 \%$ reduction in LDL-C from baseline was achieved in less than a third of individuals treated with statins. The observed mean reduction in LDL-C from baseline was $36.3 \%$ at 12 months and $39.5 \%$ at 24 months following the initiation of statin therapy. Compared with those who did not achieve the treatment target, individuals who achieved the treatment target of $\geq 50 \%$ reduction in LDL-C from baseline were predominantly women, of older age at time of $\mathrm{FH}$ diagnosis and on initiation of statin treatment; they had more severe mean pretreatment LDL-hypercholesterolaemia, and significantly higher proportions of them was treated with high-potency statins at baseline and over the 12-month period after the initiation of statins. Individuals with $\mathrm{FH}$ diagnoses, who additionally had baseline LDL-C levels at or above the FH diagnostic threshold of $7.5 \mathrm{mmol} / \mathrm{L}$, had greater 12-month and 24-month reductions in LDL-C after initiating statins, and a higher proportion of them attained the NICE guideline LDL-C treatment goal at 12 and 24 months compared with the entire cohort.

\section{Strengths and limitations}

To our knowledge, this is the first study to assess and quantify LDL-C reduction associated with statin treatment in individuals with $\mathrm{FH}$ in primary care. By using pseudonymised but comprehensively coded electronic primary care health records from a quality-assured and highly representative database, ${ }^{12}$ we were able to ascertain repeated measures of LDC-C and robustly characterise these individuals using a large sample size and a longitudinal study design. The study design minimised selection, recall and respondent bias.

We recognise certain limitations. As with all primary care electronic health records, the recording of clinical diagnosis relies on the accurate and complete recording by the general practitioner (GP) during routine consultations. ${ }^{16}{ }^{17}$ While we acknowledge the potential for FH misclassification in some individuals with hypercholesterolaemia due to secondary causes, there were no significant differences in the prevalence of comorbid conditions between those who attained and those who did not attain the LDL-C treatment goal, and thus, there is no reason to believe differential effects of such misclassification 
between the groups. It was not possible to determine whether recorded $\mathrm{FH}$ diagnoses were made following clinical assessment of FH phenotype or specialist assessment including genetic testing in secondary care or both, because there are currently no distinct codes for these in clinical systems. As there was no data on FH genetic mutation, it was not possible to assess whether there was an association between the various $\mathrm{FH}$ genetic mutation types and LDL-C treatment goal attainment. Lastly, although we explored individuals' sociodemographic and clinical characteristics in relation to their LDL-C goal attainment or non-attainment status, we did not have data on some of the factors that may be associated with treatment goal attainment such as side effects to statin treatment, non-adherence to treatment and dietary intake, and so, we were unable to explore these in our FH study cohort. Despite the above limitations, findings from our study reflect a pragmatic evaluation of LDL-C treatment goal attainment associated with statin therapy in primary care patients with $\mathrm{FH}$.

\section{Comparison with previous literature}

This is the first study to evaluate LDL-C response to statin treatment in primary care patients with FH. Suboptimal response to statins had previously been demonstrated in over half of the general population of people initiated on statins for primary prevention. ${ }^{11}$ Similarly, findings from the EURO ASPIRE V Survey in 78 centres from 16 European countries found that the recommended LDL-C target was achieved in less than half of highCVD risk individuals on lipid-lowering medication. ${ }^{18}$ In the FH population, previous studies of lipid-lowering therapy have primarily evaluated treatments among patients in lipid clinics or specialist registers, ${ }^{19-21}$ while clinical trials have assessed LDL-C reduction associated with treatment using more specialised lipid-lowering agents such as proprotein convertase subtilisin/kexin type $9 .^{22}{ }^{23}$ While it had previously been shown that less than $50 \%$ of the general population who was prescribed statins for primary CVD prevention achieved a $40 \%$ or greater reduction in LDL-C within 24 months, our study of the FH population found that $51.1 \%$ of individuals at 1 year and $56.3 \%$ of individuals at 2 years achieved this general population treatment target. This suggests that although individuals with FH may be treated better with statins than the general population, their lipid-lowering management is not optimal to the level recommended in the FH guidelines. The importance of intensive GP management of $\mathrm{FH}$ has been demonstrated in a study by Brett $e t$ al, which showed that implementing a pragmatic intervention plan by GPs, with the inclusion of statin/ ezetimibe medication \pm lifestyle advice, resulted in significant reduction in LDL-C levels. ${ }^{24}$

While there is no clear explanation for the variation in LDL-C reduction in our study population, we hypothesise that this may be partly explained by the significant differences in baseline and treatment characteristics between individuals who attained the LDL-C treatment goal and those who did not. Perhaps older adults and those with higher LDL-C at baseline are more likely to be prescribed higher-intensity statins due to their perceived increased risk of CVD, which consequently results in better LDL-C treatment outcomes.

There were no significant differences in alcohol consumption or the reported level of physical activity between statin treatment goal attainers and non-attainers, but cigarette smoke exposure was higher in those who failed to attain the LDL-C treatment goal. Although there is no clear evidence that smoking increases the levels of LDL-C, ${ }^{25}$ exposure to cigarette smoke is likely to further increase CVD risk in these individuals who fail to attain the LDL-C treatment goal.

\section{Clinical implications and conclusion}

This study provides novel knowledge of suboptimal LDL-C reduction in individuals with $\mathrm{FH}$ who are being treated with statins. With less than $40 \%$ of patients being treated with high-intensity statins and lack of attainment of the $\geq 50 \%$ LDL-C-lowering treatment goal in two-thirds of patients initiated on statin treatment, findings from this study highlight significant gaps in the $\mathrm{FH}$ patient management. Applying multifaceted approach by identifying the barriers to evidence-based FH patient care and using a strategic means to tailor effective interventions to modifiable barriers at the patient level, physician level and health system level are likely to improve implementation of evidence-based FH patient care and outcomes. ${ }^{26} 27$ Emphasis on lifestyle and dietary modification is needed to manage raised LDL-C and reduce CVD risk in individuals with FH. Intensive LDL-C lowering has clear benefits in CVD risk reduction, ${ }^{28}{ }^{29}$ so it is essential that these individuals in primary care are optimally managed and intensively monitored. Raising greater awareness of FH, in particular of the need for use of higher-potency statins, is needed in this setting.

\section{Twitter Barbara lyen @iambarbo}

Contributors BI conceptualised the study idea, designed the study, obtained ethical approvals to access data, analysed the data and drafted the first manuscript. BI, RKA, SW, JK and NQ interpreted the study findings. SW, RA, JK and $\mathrm{NQ}$ critically edited the manuscript. JK and NQ provided overall supervision of the research study. All study authors have contributed to revising, writing and finalising the final draft of the manuscript prior to submission. BI is the guarantor, accepts full responsibility for the work and the conduct of the study, had access to the data, and controlled the decision to publish.

Funding This study/project is funded by the National Institute for Health Research (NIHR) School for Primary Care Research (project reference RC48R2).

Competing interests None declared.

Patient consent for publication Not applicable.

Ethics approval Data access and ethical approval were granted by the CPRD Independent Scientific Advisory Committee (protocol number 20_093).

Provenance and peer review Not commissioned; internally peer reviewed.

Data availability statement Data are available on reasonable request. The CPRD data analysed during this study are available from the Clinical Practice Research Datalink (CPRD) (enquiries@cprd.com), but restrictions apply to the availability of these data, which were used under license for the current study and so are not publicly available. Data are however available from the authors on reasonable 
request and with permission of the CPRD Independent Scientific Advisory Committee (ISAC) (enquiries@cprd.com).

Open access This is an open access article distributed in accordance with the Creative Commons Attribution 4.0 Unported (CC BY 4.0) license, which permits others to copy, redistribute, remix, transform and build upon this work for any purpose, provided the original work is properly cited, a link to the licence is given, and indication of whether changes were made. See: https://creativecommons.org/ licenses/by/4.0/.

\section{ORCID iDs}

Barbara lyen http://orcid.org/0000-0001-9720-1180

Stephen Weng http://orcid.org/0000-0002-5281-9590

Joe Kai http://orcid.org/0000-0001-9040-9384

Nadeem Qureshi http://orcid.org/0000-0003-4909-0644

\section{REFERENCES}

1 Goldstein JL HH, Brown MS. Familial hypercholesterolemia. In: Scriver CR, Sly WS, Valle D, eds. The metabolic and molecular bases of inherited disease. 8th edition. New York: McGraw-Hill, Inc, 2001: 2863-913.

2 Austin MA, Hutter CM, Zimmern RL, et al. Genetic causes of monogenic heterozygous familial hypercholesterolemia: a huge prevalence review. Am J Epidemiol 2004;160:407-20.

3 Besseling J, Hovingh GK, Huijgen R, et al. Statins in Familial Hypercholesterolemia: Consequences for Coronary Artery Disease and All-Cause Mortality. J Am Coll Cardiol 2016;68:252-60.

4 Versmissen J, Oosterveer DM, Yazdanpanah M, et al. Efficacy of statins in familial hypercholesterolaemia: a long term cohort study. BMJ 2008;337, :a2423.

5 National Institute for Health and Care Excellence. Familial hypercholesterolaemia: identification and management National Institute for health and care excellence. NICE clinical guideline (CG71). Available: https://www.nice.org.uk/guidance/cg71

6 Mach F, Baigent C, Catapano AL, et al. 2019 ESC/EAS guidelines for the management of dyslipidaemias: lipid modification to reduce cardiovascular risk. Eur Heart J 2020;41:111-88.

7 Khatib R, Neely D. Summary of national guidance for lipid management for primary and secondary prevention of CVD." [Online]. Available: https://www.england.nhs.uk/aac/wp-content/ uploads/sites/50/2020/04/lipid-management-pathway-guidance.pdf

8 Boekholdt SM, Hovingh GK, Mora S, et al. Very low levels of atherogenic lipoproteins and the risk for cardiovascular events: a meta-analysis of statin trials. J Am Coll Cardiol 2014;64:485-94.

9 Reiner Z. Resistance and intolerance to statins. Nutr Metab Cardiovasc Dis 2014;24:1057-66.

10 Chasman DI, Giulianini F, MacFadyen J, et al. Genetic determinants of statin-induced low-density lipoprotein cholesterol reduction: the justification for the use of statins in prevention: an intervention trial evaluating rosuvastatin (JUPITER) trial. Circ Cardiovasc Genet 2012;5:257-64.

11 Akyea RK, Kai J, Qureshi N, et al. Sub-optimal cholesterol response to initiation of statins and future risk of cardiovascular disease. Heart 2019;105:975-81.

12 Herrett E, Gallagher AM, Bhaskaran K, et al. Data resource profile: clinical practice research Datalink (CPRD). Int J Epidemiol 2015;44:827-36.

13 Law MR, Wald NJ, Rudnicka AR. Quantifying effect of statins on low density lipoprotein cholesterol, ischaemic heart disease, and stroke: systematic review and meta-analysis. BMJ 2003;326, :1423.
14 National Institute for Health and Care Excellence. Cardiovascular disease: risk assessment and reduction, including lipid modification. Clinical guideline [CG181]. Available: https://www.nice.org.uk/ guidance/cg181/chapter/1-Recommendations\#lipid-modificationtherapy-for-the-primary-and-secondary-prevention-of-cvd-2 [Accessed 24 May 2021].

15 National Institute for Health and Care Excellence. Lipid modification - CVD prevention. Available: https://cks.nice.org.uk/topics/lipidmodification-cvd-prevention/management/lipid-therapy-primaryprevention-of-cvd/

16 Shephard E, Stapley S, Hamilton W. The use of electronic databases in primary care research. Fam Pract 2011;28:352-4.

17 Manuel DG, Rosella LC, Stukel TA. Importance of accurately identifying disease in studies using electronic health records. BMJ 2010;341, :c4226.

18 Kotseva K, De Backer G, De Bacquer D, et al. Primary prevention efforts are poorly developed in people at high cardiovascular risk: a report from the European Society of cardiology EURObservational research programme EUROASPIRE V survey in 16 European countries. Eur J Prev Cardiol 2020:370-9.

19 Schmidt N, Dressel A, Grammer TB, et al. Lipid-modifying therapy and low-density lipoprotein cholesterol goal attainment in patients with familial hypercholesterolemia in Germany: the CaReHigh registry. Atherosclerosis 2018;277:314-22.

20 Rizos CV, Elisaf MS, Skoumas I, et al. Characteristics and management of 1093 patients with clinical diagnosis of familial hypercholesterolemia in Greece: data from the Hellenic familial hypercholesterolemia registry (HELLAS-FH). Atherosclerosis 2018;277:308-13.

21 Climent E, Marco-Benedí V, Benaiges D, et al. Impact of statin therapy on LDL and non-HDL cholesterol levels in subjects with heterozygous familial hypercholesterolaemia. Nutr Metab Cardiovasc Dis 2021;31:1594-603.

22 Kastelein JJP, Ginsberg HN, Langslet G, et al. Odyssey FH I and FH II: 78 week results with alirocumab treatment in 735 patients with heterozygous familial hypercholesterolaemia. Eur Heart $J$ 2015;36:ehv370-3.

23 Raal FJ, Stein EA, Dufour R, et al. Pcsk9 inhibition with evolocumab (AMG 145) in heterozygous familial hypercholesterolaemia (RUTHERFORD-2): a randomised, double-blind, placebo-controlled trial. Lancet 2015;385:331-40.

24 Brett T, Chan DC, Radford J, et al. Improving detection and management of familial hypercholesterolaemia in Australian general practice. Heart 2021;107:1213-9.

25 Gepner AD, Piper ME, Johnson HM, et al. Effects of smoking and smoking cessation on lipids and lipoproteins: outcomes from a randomized clinical trial. Am Heart J 2011;161:145-51.

26 Nieuwlaat R, Schwalm J-D, Khatib R, et al. Why are we failing to implement effective therapies in cardiovascular disease? Eur Heart $J$ 2013;34:1262-9.

27 Uchmanowicz I, Hoes A, Perk J, et al. Optimising implementation of European guidelines on cardiovascular disease prevention in clinical practice: what is needed? Eur J Prev Cardiol 2021;28:426-31.

28 Cholesterol Treatment Trialists' (CTT) Collaboration, Baigent C, Blackwell L, et al. Efficacy and safety of more intensive lowering of LDL cholesterol: a meta-analysis of data from 170,000 participants in 26 randomised trials. Lancet 2010;376:1670-81.

29 McCormack T, Dent R, Blagden M. Very low LDL-C levels may safely provide additional clinical cardiovascular benefit: the evidence to date. Int J Clin Pract 2016;70:886-97. 